\title{
Fungal retinitis: a case of Torulopsis glabrata infection treated with miconazole
}

\author{
ROBIN B. FITZSIMONS, ${ }^{1}$ MARYANN D. NICHOLLS, ${ }^{1}$ F. A. BILLSON, ${ }^{2}$ \\ T. I. ROBERTSON, ${ }^{1}$ AND P. HERSEY ${ }^{1}$ \\ From the ${ }^{1}$ Division of Medicine, Sydney Hospital, Macquarie Street, Sydney 2000, and \\ ${ }^{2}$ The University of Sydney, Department of Clinical Ophthalmology, Sydney Eye Hospital, \\ Sir John Young Crescent, Woolloomooloo, NSW, Australia 2011
}

SUMmARY Presumptive embolic chorioretinal Torulopsis glabrata infection is described in a patient who had received prolonged intravenous antibiotic therapy. The ocular findings are compared and contrasted with embolic lesions due to Candida albicans. The patient was treated for 6 weeks with intravenous miconazole. During this time there was shrinkage of the ocular lesions, some improvement in vision and abolition of fungaemia. Improvement in tests of immune function during treatment suggests that an early immunological deficit was secondary to the infection. Intravenous miconazole is a relatively nontoxic alternative to amphotericin and deserves further evaluation in the treatment of ocular mycosis.

Fungal infections occur in the immunologically compromised host and are a well recognised complication of intravenous antibiotic therapy ${ }^{1-5}$ and intravenous drug addiction. ${ }^{6-8}$ Embolic ocular manifestations of systemic fungal infection generally present as chorioretinal lesions with subsequent anterior spread; Candida albicans is the organism most often implicated. ${ }^{910}$ Torulopsis glabrata may also cause fungaemia in similar circumstances, but retinal torulopsis has not been described. Amphotericin, the drug commonly used in the treatment of the ocular mycoses, may cause severe renal, hepatic, and bone marrow toxicity. Fluorocytosine is a less toxic alternative, but the organism may become rapidly resistant when it is prescribed alone. ${ }^{11}$ Miconazole is a new drug being used in the treatment of systemic mycosis. It does not appear to have the serious adverse effects of amphotericin.

This paper describes a patient with chorioretinitis associated with Torulopsis glabrata fungaemia and the response of both ocular and systemic infection to intravenous miconazole.

\section{Case report}

A 31-year-old woman was referred to Sydney Hospital on 29 May 1979 with a 2-month history of blurred vision in the left eye, deteriorating further in the week prior to admission. In December 1978

Correspondence to Dr F. A. Billson. she allegedly fell on a cup and deeply lacerated the left foot. Recurrent infection followed which necessitated prolonged intravenous antibiotic therapy. She was febrile on admission, and there was chronic inflammation with suppuration of the soft tissue of the dorsum of the foot. Regional lymph nodes were not enlarged.

Visual acuity in the right eye was $6 / 9$ and funduscopy and fluorescein angiography were normal. Vision in the left eye was reduced to appreciation of hand movements in the peripheral field with no vision in the right upper quadrant. There was a minor vitreous haze. The optic disc was elevated, and there was a white circumscribed lesion surrounded by satellite bodies in the lower temporal quadrant (Fig. 1a). There was neovascularisation as well as traction of the retina, with gross distortion of the retinal vasculature and the macula.

Fluorescein angiography revealed a retinal mass immediately inferior to the macula surrounded by radiating capillaries, which fluoresced early. In the later phases of the angiogram there was gross leakage of dye from these capillaries, as well as from the temporal side of the disc (Fig. 2). These radiating vessels were interpreted as neovascularisation because they were anterior to the normal retinal capillaries in stereoscopic views.

The wound on the foot was explored and Torulopsis glabrata was cultured from debrided tissue. It was also grown on Sabouraud's medium from duplicate blood culture bottles. Cultures taken from 

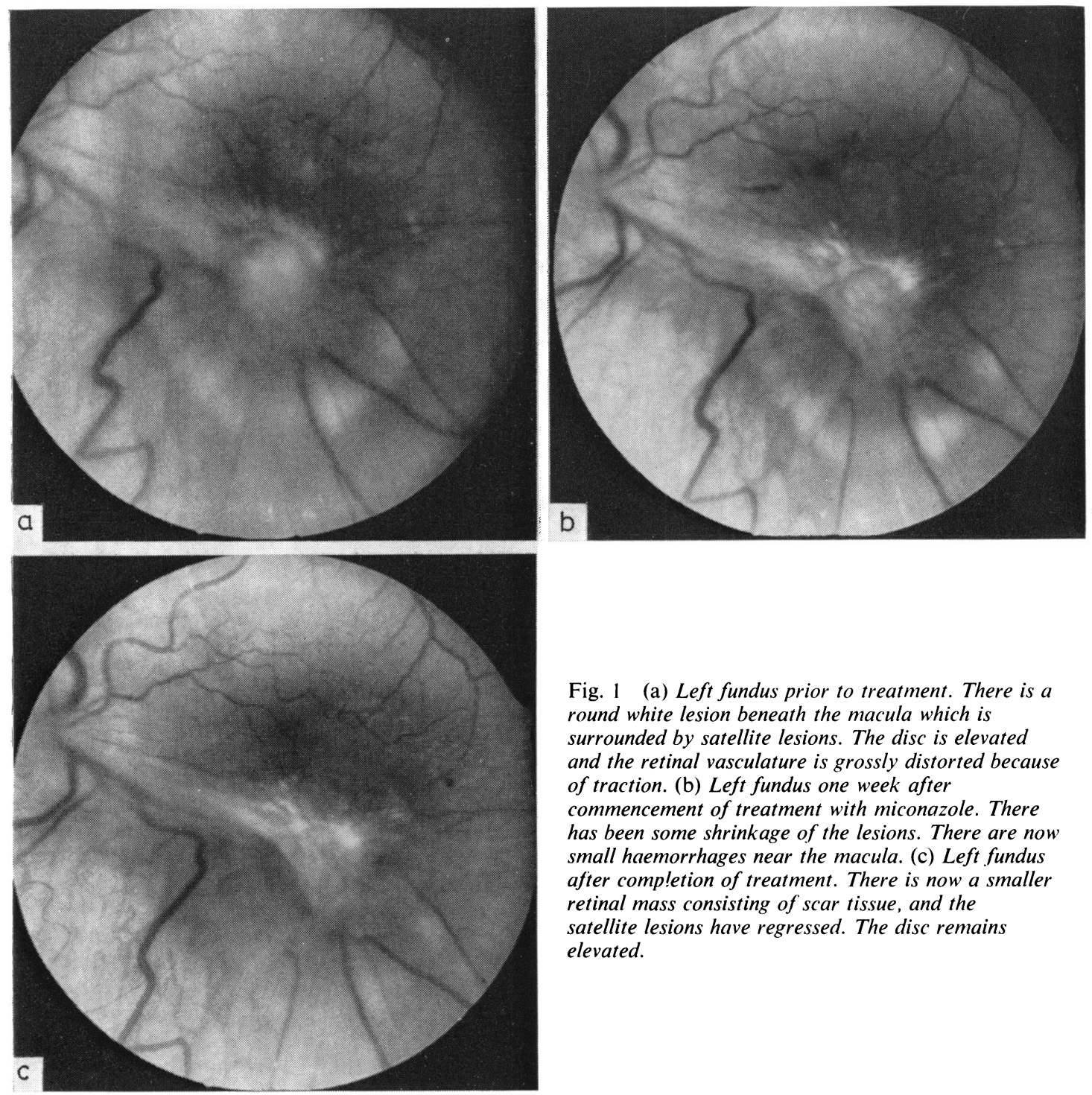

the vagina and throat swabs showed a light growth of Candida albicans. Antibodies to Torulopsis glabrata were present in a titre of $1: 320$ and to Candida albicans in a titre of $1: 80$. The haemoglobin was $11.7 \mathrm{~g} / \mathrm{dl}$ and the leucocyte count $8 \times 10^{9} / \mathrm{l}$. There was slight eosinophilia $\left(1.26 \times 10^{9} / \mathrm{l}\right)$ and lymphocytopenia $\left(0.980 \times 10^{9} / 1\right)$. The erythrocyte sedimentation rate was $35 \mathrm{~mm} / \mathrm{h}$. The glucose tolerance test and tests of renal and hepatic function were normal.

The patient was initially thought to have had atypical ocular candidiasis. The structural changes at the macula suggested that prognosis for vision was poor. It was decided to treat the patient with
Fig. 1 (a) Left fundus prior to treatment. There is a round white lesion beneath the macula which is surrounded by satellite lesions. The disc is elevated and the retinal vasculature is grossly distorted because of traction. (b) Left fundus one week after commencement of treatment with miconazole. There has been some shrinkage of the lesions. There are now small haemorrhages near the macula. (c) Left fundus after completion of treatment. There is now a smaller retinal mass consisting of scar tissue, and the satellite lesions have regressed. The disc remains elevated.

miconazole because of the known toxicity of amphotericin. Pending results of culture, treatment was commenced with miconazole by daily infusion of $1000 \mathrm{mg}$ in $5 \%$ dextrose in water over 2 hours. This therapy was continued when blood cultures showed the presence of Torulopsis glabrata. Following the determination of the minimum inhibitory concentration $(\mathrm{MIC}=0.5 \mathrm{mcg} / \mathrm{ml}$ ) and minimum fungicidal concentration $(\mathrm{MFC}=20 \mathrm{mcg} / \mathrm{ml})$ of miconazole for this organism the daily dose was increased to $2400 \mathrm{mg}$ as twice daily infusions over 1-2 hours delivered via a Raimondi catheter, which remained in place throughout the 6 weeks of therapy. The catheter has distal unidirectional 


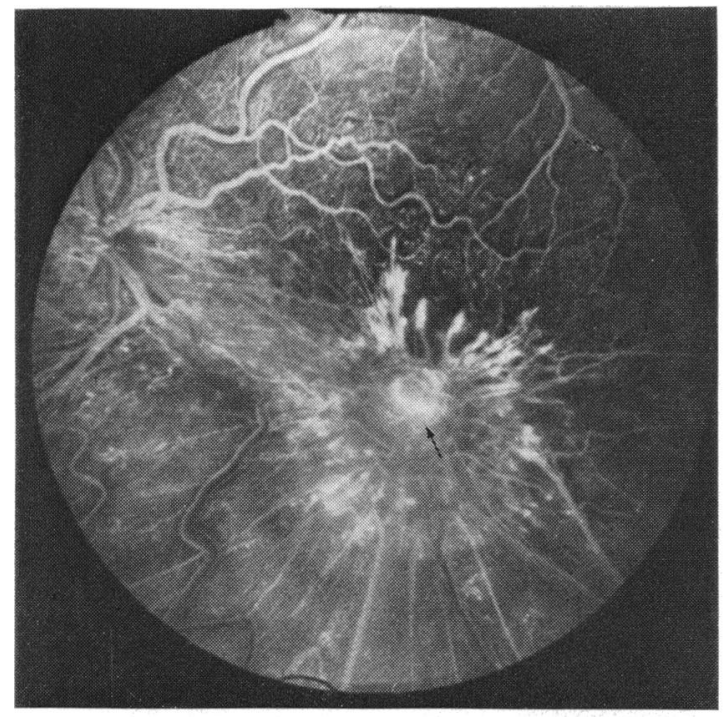

Fig. 2 Fluorescein angiogram of left fundus. The retinal mass is surrounded by radiating capillaries which fluoresced early. Stereoscopic views demonstrated capillaries anterior to the normal retinal vasculature. The pattern of fluorescence in the centre of the lesion was suggestive of chorioretinal anastomosis (see arrow).

valves, and is relatively resistant to thrombosis and infection. It was inserted because of poor venous access, despite reservations regarding the risks of prolonged central venous lines. ${ }^{12}$

One week after commencement of therapy small vertical haemorrhages were noted above the main lesion in the region of the macula (Fig. 1b). During the 6 weeks of treatment vision improved to $6 / 60$, the vitreous cleared completely, and the satellite lesions regressed. The main embolic lesion also decreased in size significantly. Since discharge from hospital on 21 July 1979 the appearance of this scarred lesion (Fig. 1c) has remained static. Elevation of the disc due to traction has persisted. During treatment a pruritic rash developed which was treated with antihistamines. Blood cultures on 5 separate occasions, the latest on 8 October 1979, during and after completion of treatment have been consistently negative.

Immune function tests before treatment showed depressed cell-mediated immunity. $T$ cell numbers (E rosette formation), as identified by their ability to bind sheep erythrocytes to their surfaces, were reduced to $34 \%$ of the total lymphocytes. B cell numbers were relatively increased to $58 \%$. With treatment $\mathrm{E}$ rosette formation increased to $65 \%$ and $B$ cells decreased to $31 \%$. Antigenic skin tests for cell-mediated response using saline, candida and purified protein derivative (PPD) were all negative on 1 June 1979 , but a $4.0 \mathrm{~mm}$ area of induration to streptokinase-streptodornase (SKSD) developed. These tests improved with antifungal treatment to $6.0 \mathrm{~mm}$ induration with PPD and 20.0 $\mathrm{mm}$ to SKSD. There was persistent anergy to candida. The response of the patient's $T$ lymphocytes to phytohaemagglutinin (PHA) was abnormally low prior to treatment and returned to normal during treatment. No circulating immune complexes were detected by the ${ }^{125} \mathrm{I} \mathrm{Clq}$ precipitation test. Immune globulins were not reduced.

The electrocardiogram was normal and echocardiography showed no evidence of valvular disease. Fasting serum triglycerides and serum cholesterol were respectively $2.37 \mathrm{mmol} / 1$ (normal less than $1.70 \mathrm{mmol} / \mathrm{l}$ ) and $6.5 \mathrm{mmol} / \mathrm{l}$ (normal 3.9-6.7 mmol/l) initially and rose during treatment to $12.4 \mathrm{mmol} / \mathrm{l}$ and $22 \cdot 12 \mathrm{mmol} / \mathrm{l}$ respectively. Two months after the completion of therapy these figures had fallen to $2.40 \mathrm{mmol} / \mathrm{l}$ and $6.3 \mathrm{mmol} / \mathrm{l}$.

\section{Discussion}

The clinical findings of a white retinal mass, with surrounding satellite lesions, seen in the context of chronic intravenous antibiotic administration or drug addiction, are strongly suggestive of embolic ocular fungal infection. In the present case the patient gave a history of trauma and prolonged intravenous antibiotic therapy, but the possibility that drug addiction was also a factor has not been dismissed. She has subsequently presented to 2 other hospitals in Sydney and given totally different accounts of her symptoms, and a Munchausen syndrome appears likely. ${ }^{13}$

Candida albicans was grown from throat and vaginal swabs, but the subsequent confirmation of deep wound infection and fungaemia with Torulopsis glabrata make it highly probable that this organism was responsible for the retinal lesions. Vitreous aspiration and culture ${ }^{7}$ were not thought justified in view of only minor vitreous haze.

Embolic ocular candidiasis is characterised by fluffy white inner retinal lesions, which rapidly spread anteriorly to cause severe vitreous opacification, which in chronic cases may obscure the retinal changes. ${ }^{56}$ Fluorescein angiography may reveal progressive fluorescence of retinal lesions, or areas of early hypofluorescence in areas of choroiditis, ${ }^{4} 7$ but new vessel formation is not described.

The clinical features in our case were different. Retinal vascularity with neovascularisation was striking. Vitreous involvement was minor, even though the clinical history and neovascularisation were consistent with a disease process extending over months. 
Torulopsis glabrata is a yeast-like fungus, which is a normal saprophyte in man. ${ }^{14}$ It is related to candida, but can be differentiated from it by colonial appearance, lack of true mycelial growth, and by fermentation reactions. It is an opportunistic pathogen in immunosuppressed patients, and may cause fungaemia, ${ }^{121516}$ systemic disease, ${ }^{17-19}$ and wound infection, ${ }^{20}$ including infection following keratoplasty. ${ }^{21}$ Anterior chamber infection with both Aspergillus flavus and Torulopsis glabrata in a drug addict has been described by Elliott et al. ${ }^{8}$

The response of the patient's fungaemia and retinitis to miconazole is important. Amphotericin, which is generally effective in treating ocular mycoses, ${ }^{282122}$ has potentially serious adverse effects which can complicate the already difficult course of an illness in debilitated and immune suppressed patients. The absence of these side effects is a major advantage for miconazole. ${ }^{23}$

Miconazole is an imidazole derivative with a broad spectrum of activity against Gram-positive cocci and most fungal diseases, including candidiasis, asperigillosis, histoplasmosis, and infections with torulopsis. In low doses it is fungistatic, and therefore treatment may need to be prolonged to ensure adequate eradication of the fungus. At very high doses it is fungicidal. It penetrates well into most infected tissues, including vitreous humour, ${ }^{23}$ but penetration into the cerebrospinal fluid is poor. Minor side effects include pruritus, rash, chills, phlebitis, and gastrointestinal symptoms, but no major adverse reactions have been reported to date. A transitory increase in blood lipids may occur, as in the present case, because of the lipid solvent (polyethoxylated castor oil) present in the parenteral form of the drug.

Initial investigation of the immune status of this patient revealed marked abnormalities of the cellmediated response, as indicated by reduced $\mathrm{E}$ rosette formation, anergy of skin tests to common recall antigens, and absent response to PHA. During treatment most of these tests returned to normal, indicating that the fungal infection was most probably the cause of the immunological abnormalities. These abnormalities may have been due in part to the development of suppressor cells against the immune response to the fungus. ${ }^{24} \mathrm{~A}$ more subtle defect in the ability of the patient's immune system to respond to fungal infection may still exist.

It would appear that miconazole represents a potentially useful alternative to amphotericin in the treatment of ocular torulopsis.

We thank Dr F. Jennis, microbiologist, Sydney Hospital, Mr P. Crea for advice concerning the Raimondi catheter,
Dr J. Pollard for permission to report this case, and Mr G. Congdon for the fundus photography.

\section{References}

1 Edwards JE, Foos RY, Montgomerie JZ, Guze LB. Ocular manifestations of Candida septicemia: Review of seventy-six cases of hematogenous Candida endophthalmitis. Medicine (Baltimore) 1974; 53: 47-75.

2 Griffin JR, Pettit TH, Fishman LS, Foos RY. Bloodborne Candida endophthalmitis. A clinical and pathological study of 21 cases. Arch Ophthalmol 1973; 89: 450-6.

3 Meyers BR, Lieberman TW, Ferry AP. Candida endophthalmitis complicating candidaemia. Ann Intern Med 1973; 79: 647-53.

4 Robertson DM, Riley FC, Hermans PE. Endogenous candida oculomycosis. Arch Ophthalmol 1974; 91: 33-8.

5 Weinstein AJ, Johnson EH, Moellering RC. Candida endophthalmitis. A complication of candidemia. Arch Intern Med 1973; 132: 749-52.

6 Getnick RA. Rodrigues MM. Endogenous fungal endophthalmitis in a drug addict. Am J Ophthalmol 1974; 77: 680-3.

7 Stern GA, Fetkenhour CL, O'Grady RB. Intravitreal amphotericin B treatment of candida endophthalmitis. Arch Ophthalmol 1977; 95: 89-93.

8 Elliott JH, O'Day DM, Gutow GS, Podgorski SF, Akrabawi P. Mycotic endophthalmitis in drug abusers. Am J Ophthalmol 1979; 88: 66-72.

9 Francois P, Turut P, Guilbert F. Les candidoses retiniennes. Arch Ophtalmol (Paris) 1974; 34: 863-77.

10 Fleming KO. Candida albicans abscess of retina. Can J Ophthalmol 1972; 7: 132-5.

11 Bennett JE. Flucytosine. Ann Intern Med 1977; 86: 31922.

12 Rodrigues RJ, Shinya H, Wolff WI, Puttlitz D. Torulopsis glabrata fungemia during prolonged intravenous alimentation therapy. $N$ Engl J Med 1971; 284: 540-1.

13 Asher R. Munchausen's syndrome. Lancet 1951; i: 339-41.

14 Sternderup A, Pedersen GT. Yeasts of human origin. Acta Pathol Microbiol Scand 1962; 54: 462-72.

15 Grimley PM, Wright LD Jr, Jennings AE. Torulopsis glabrata infection in man. Am J Clin Pathol 1965; 43: 216-23.

16 Marks MI, Langston C, Eikhoff TC. Torulopsis glabrata -an opportunistic pathogen in man. $N$ Engl J Med 1970; 283: $1131-5$.

17 Lees AW, Rao SS, Garrett JA, Boot PA. Endocarditis due to Torulopsis glabrata. Lancet 1971; i: 943-4.

18 Minkowitz S, Koffler D, Zak FG. Torulopsis glabrata septicemia. Am J Med 1963; 34: 252-5.

19 Aisner J, Schimpff SC, Sutherland JC, Young VM, Wiernik PH. Torulopsis glabrata infections in patients with cancer. Am J Med 1976; 61: 23-8.

20 Steer PL, Marks MI, Klite PD, Eickhoff TC. 5-Fluorocytosine; an oral antifungal compound. Ann Intern Med 1972; 76: 15-22.

21 Larsen PA, Lindstrom RL, Doughman DJ. Torulopsis glabrata endophthalmitis after keratoplasty with an organcultured cornea. Arch Ophthalmol 1978; 96: 1019-22.

22 Jones BR. Principles in the management of oculomycosis. Am J Ophthalmol 1975; 79: 719-51.

23 Symoens J. Clinical and experimental evidence on miconazole for the treatment of systemic mycosis: a review. Proc $R$ Soc Med 1977; 70 (Suppl 1): 4-8.

24 Stobo JD, Sigrun P, Van Scoy RE, Hermans PE. Suppressor thymus-derived lymphocytes in fungal infection. J Clin Invest 1976; 57: 319-28. 